\title{
ESTIMATIVA DE CONCENTRAÇÃO DE SEDIMENTOS EM SUSPENSÃO A PARTIR DE IMAGENS LANDSAT 8 EM PCHS NO RIO IVAÍ-RS
}

\author{
ESTIMATE OF SUSPENDED SEDIMENT CONCENTRATION IN SHPPS IN THE RIVER IVAÍ-RS \\ FROM LANDSAT 8 IMAGES
}

\section{Daniela Wancura Barbieri PEIXOTO' ${ }^{1}$, Laurindo Antonio GUASSELLI ${ }^{1}$, Waterloo PEREIRA FILHO ${ }^{2}$}

1Programa de Pós Graduação em Sensoriamento Remoto / Universidade Federal do Rio Grande do Sul. - Campus do Vale. Porto Alegre - RS - Brasil. Emails: daniwbarbieri@gmail.com; laurindo.guasselli@ufrgs.br

${ }^{2}$ Programa de Pós-Graduação em Geografia / Universidade Federal de Santa Maria, Departamento de Geociências, Campus da UFSM. Santa Maria - RS, Brasil. Email: waterloopf@gmail.com

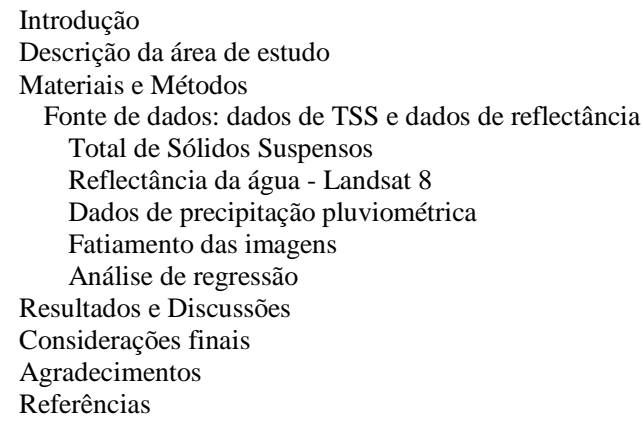

RESUMO - Nas últimas décadas o uso de sensores remotos tem ganhado destaque na estimativa e na análise da dinâmica da distribuição espacial da concentração de sedimentos suspensos, com potencial para o monitoramento de rios e reservatórios. Assim este trabalho objetiva avaliar o uso de Imagens Landsat 8 (banda 4, com faixa espectral de 640 - $670 \mathrm{~nm}$ ) para estimar a concentração de sedimentos em suspensão medidas nas três PCHs no rio Ivaí, RS. Foram utilizadas 4 imagens em reflectância disponibilizadas pela USGS (https://earthexplorer.usgs.gov) e 4 dados de TSS coletados em campo nas três PCHs em estudo afim de gerar o modelo matemático, formando 3 pontos por campo, totalizando 12 pontos de correlações. Os valores de reflectância da banda 4, com centro de banda no comprimento de onda de $655 \mathrm{~nm}$, foram correlacionados com dados de TSS. Utilizou-se o modelo exponencial que obteve o melhor ajuste de $\mathrm{R}^{2}(0,84)$ com a equação $\mathrm{y}=1,6865 \mathrm{e}^{0,1058 x}$. Verificou-se que as imagens Landsat 8 (banda 4) mostraram bom desempenho em condições específicas do ambiente aquático caracterizando a deposição dos sedimentos devido ao efeito cascata.

Palavras-chave: Reflectância; Sólidos Suspensos; Reservatórios; Landsat 8; Estimativa.

\begin{abstract}
In the last decades the use of remote sensor has been gaining distinction on both the estimate and the dynamics analysis of spatial distribution of the suspended sediment concentration, having potential to be used on rivers and reservoirs. Thus this work aims to evaluate the usage from the Landsat 8 Images (band 4, with spectral range $640-670 \mathrm{~nm}$ ) in order to estimate the suspended sediment concentration on the three SHPPs (Small Hydroelectric Power Plant) of the river Ivaí, RS. Four reflectance images were used and made available by USGS (www.earthexplorer.usgs.gov) and 4 TSS field collected data in three SHPPs under study to produce the mathematical model, forming 3 points per field for a total amount of 12 correlated points. The reflectance values from the band 4 , with band center of $655 \mathrm{~nm}$ of wavelength, were correlated to the TSS data. The exponential model that presented the best setting of $\mathrm{R}^{2}(0.84)$ was used to the equation $\mathrm{y}=1,6865 \mathrm{e}^{0,1058 \mathrm{x}}$. It was verified that the images from Landsat 8 (band 4 ) display a fine development in specific aquatic environment conditions, outlining the sediment deposition due to ripple effect.
\end{abstract}

Keywords: Reflectance; Suspended Solids; Reservoirs; Landsat 8; Estimate.

\section{INTRODUÇÃO}

O total de sedimentos em suspensão (TSS) é um dos principais fatores que afetam a penetração da luz em ambiente aquático. A influência do sedimento em um ecossistema aquático pode ser permanente e potencialmente prejudicial em muitos casos, pois altas concentrações de TSS afetam diretamente a qualidade da água (Tian et al., 2014).

Investigadores relatam forte correlação positiva entre sedimentos suspensos e a reflectância, e afirmam que métodos de amostragem são muitas vezes insuficientes em termos de cobertura espacial, temporal e também econômica para se obter resultados estatisticamente significativos. No entanto, o sensoriamento remoto permite que a análise da qualidade da água seja possível pois permite uma coleção de dados sobre uma grande área, ao mesmo tempo, e proporciona uma melhor cobertura de dados, facilitando as autoridades de gestão métodos eficazes para monitoramento (Zhimin et al., 1992; Jinling et al., 2015; Wang \& Lu, 2010; Tian et al., 2014).

Existem muitos casos de sucesso na literatura em que o TSS foi estimado de dados de sensores remotos nos últimos 40 anos. Tian et al. (2014) destacam que na década de 70 pesquisadores construíram um modelo estatístico quantitativo 
para a recuperação de sedimentos em suspensão usando digitalizador multiespectral (MSS). Posteriormente os sensores MODIS, MERIS, e SPOT Landsat TM / ETM + têm sido utilizados para avaliar a concentração do TSS em diferentes áreas de estudo.

Desta forma, Lin et al. (2012) afirmam que nos últimos 10 anos estes estudos se intensificaram favorecendo a detecção remota por satélite, tornando possível monitorar o transporte de sedimentos e analisar o destino e distribuição dos sólidos em suspensão na água oferecendo uma visão instantânea da concentração de sedimentos.

Montanher et al. (2014), afirmam que técnicas de sensoriamento remoto foram utilizadas para estimar os parâmetros de qualidade da água em todo o mundo, a partir do qual os dados de material em suspensão é o mais utilizado obtendo sucesso.

Segundo Lin et al. (2012) os algoritmos de estimativa de material em suspensão incluem três principais categorias: i) o modelo empírico; ii) o modelo físico; iii) o modelo semi-analítico. Os dois últimos de base física, mas precisam de dados de observação e avaliados in situ, como por exemplo: TSS, clorofila, dados espectrorradiométricos. Apesar de o modelo empírico carecer de uma base física, ele tem uma forma simples e só precisa de dados de TSS e Reflectância, por isso é mais disponível e operacional.

Portanto, o sensoriamento remoto tem o potencial para monitoramento da dinâmica e da distribuição espacial da concentração de sedimentos suspensos. É necessário, entretanto, entender a reflectância em rios e reservatórios. Entender a relação da reflectância espectral da água com os fenômenos/características de uma bacia hidrográfica facilita ao pesquisador estimar a distribuição e a concentração de sólidos em suspensão sobre a água, sejam elas continentais, reservatórios, lagos ou rios.

Neste sentido, este trabalho aborda estimativas de TSS em pequenas áreas de estudo, ou seja, em Pequenas Centrais Hidrelétricas (PCH), pois o desenvolvimento energético brasileiro é caracterizado pelas grandes centrais hidrelétricas para que abasteça grandes centros, devido a isto, os municípios isolados ou áreas rurais muitas vezes são negligenciados devido ao custo para interligação dessas localidades.

No Brasil, uma usina hidrelétrica pode ser classificada em dois principais tipos: $\mathrm{PCH}$, usina de pequeno porte cuja capacidade instalada seja superior a $1 \mathrm{MW}$ e inferior a 30 MW com reservatório inferior a $3 \mathrm{~km}^{2}$ e GCH's, ou grandes centrais hidrelétricas que produzem acima de 30 MW (Resolução ANEEL N. ${ }^{\circ}$ 394/98) (BRASIL, 1998).

Neste contexto, a implantação de PCHs é uma opção para suprir energia em municípios distantes e sem conexão ao sistema, como também, permite um menor impacto por alagarem pequenas extensões de terras. Todavia as PCHs são associadas à geração de menores impactos se comparada às grandes usinas hidrelétricas, uma vez que, normalmente possuem barragens menores e consequentemente alagam áreas menores.

Assim este trabalho objetiva avaliar o uso de Imagens Landsat 8 (banda 4) para estimar a concentração de sedimentos em suspensão medida em três PCHs no rio Ivaí, RS.

\section{DESCRIÇÃO DA ÁREA DE ESTUDO}

Com a finalidade de geração de energia elétrica foram construídos PCHs no curso do rio Ivaí, no município de Júlio de Castilhos-RS. A partir da década de 50 foi inaugurada a primeira PCH do Ivaí (1950), hoje conhecida como Cascata do Ivaí. Após foram construídas mais duas, uma a sua montante PCH Ernesto Jorge Dreher, em funcionamento desde 2009 e outra a sua jusante denominada Henrique Kotzian, operando desde 2011.

A primeira $\mathrm{PCH}$ da série em cascata Eng. Ernesto Dreher possui uma potência instalada de $17.870 \mathrm{~kW}$ com uma vazão de $33,78 \mathrm{~m}^{3} / \mathrm{s}$, energia capaz de abastecer 124 mil consumidores.
A área de drenagem do local onde está localizada a PCH Ernesto Jorge Dreher é de $1.552 \mathrm{~km}^{2}$. A segunda da série é a menor, possui uma potência de $1,7 \mathrm{~kW}$, e a terceira tem uma potência instalada de $13.000 \mathrm{~kW}$ e uma vazão de $48,25 \mathrm{~m}^{3} / \mathrm{s}$, sua área de drenagem é de $1.640 \mathrm{~km}^{2}$, com uma profundidade máxima de aproximadamente $38 \mathrm{~m}$ (CMSAPAR, 2017). A Figura 1 mostra a localização da área de estudo.

A Bacia Hidrográfica do rio Ivaí engloba sete municípios, porém dois municípios são atingidos pelas PCHs. Na margem direita, Júlio de Castilhos e na margem esquerda o município de Salto do Jacuí. A Figura 1 mostra a localização da área. 


\section{Localização da área de estudo: PCHs no Rio Ivaí}
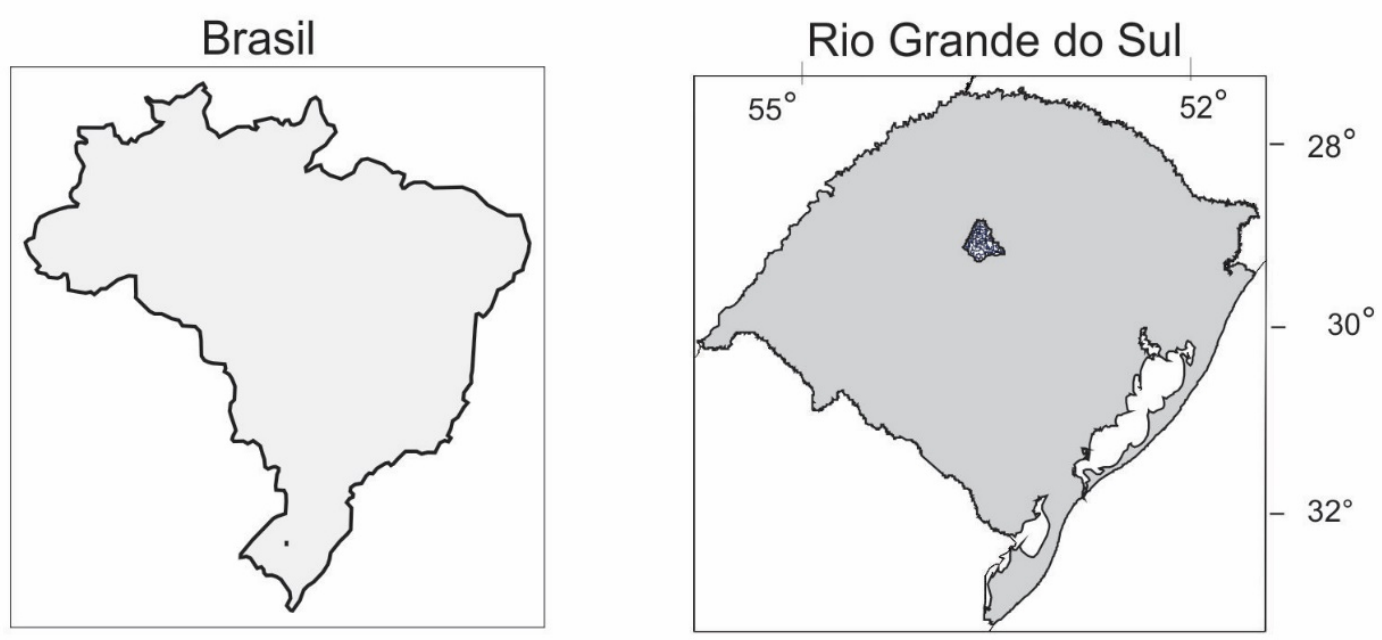

$53^{\circ} 25^{\prime} 0^{\prime \prime} \mathrm{W}$

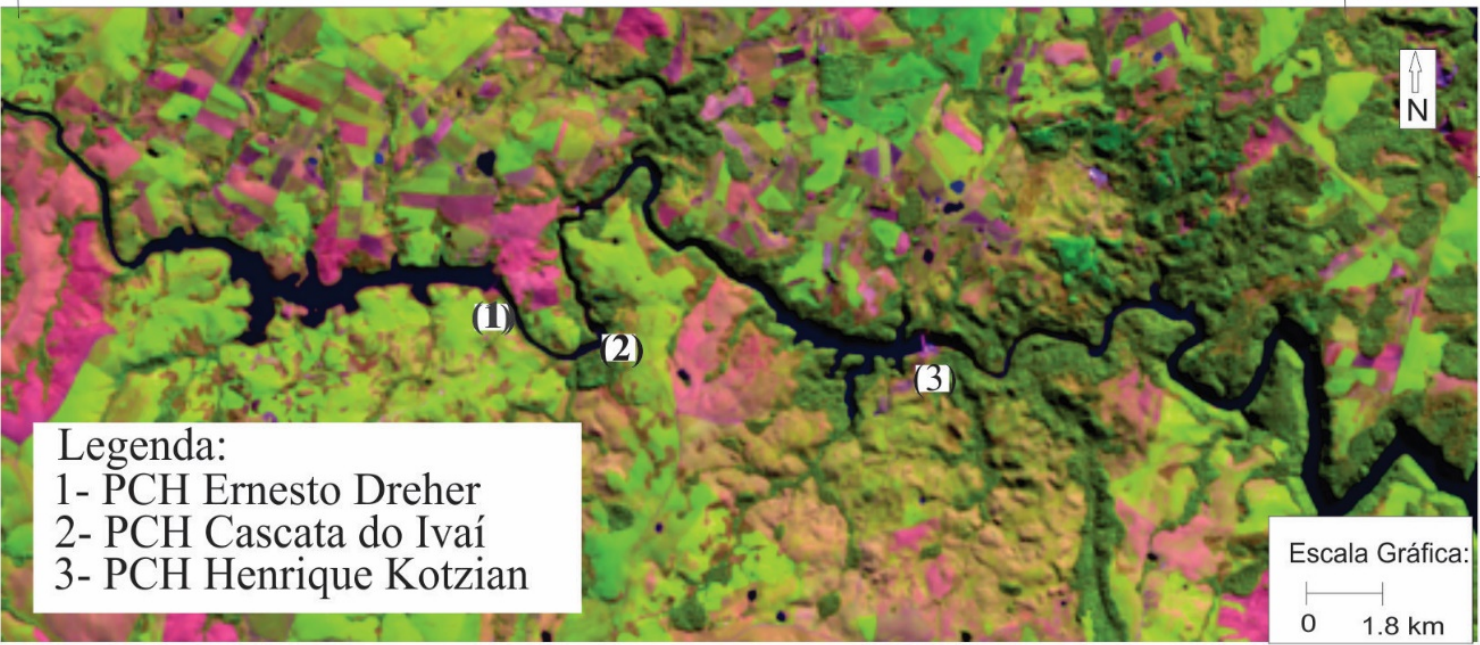

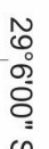

Imagem satélite Landsat 8- OLI (RGB- 6;5;4) 08-06-2105

Sistema de projeção Sirgas 2000

Figura 1 - Localização da bacia de captação das PCHs do Ivaí, RS - Brasil.

O clima da região Sul do Brasil tem papel fundamental nas atividades agrícolas desenvolvidas como também na inserção de reservatórios devido ao relevo e clima favorável. O clima predominante é o subtropical, sendo este a transição entre o clima tropical e o clima temperado das médias latitudes.

Nesta região, a precipitação pluviométrica é influenciada por fenômenos atmosféricos, entre os mais importantes, pode-se citar a passagem de sistemas frontais (encontro de massa de ar frio e quente), que são responsáveis por grande parte dos totais pluviométricos registrados (Oliveira, 1986; Sartori, 2000). No Estado do Rio Grande do Sul a precipitação média situase entre 1.299 e $1.500 \mathrm{~mm}$ e, ao norte a média está entre 1.500 e 1.800 mm (Sartori, 1993). A região Sul do Brasil apresenta uma variabilidade da precipitação tais como distribuição, intensidade e volume das chuvas de um determinado local devido a fatores geográficos associados à dinâmica da circulação atmosférica regional e ao fenômeno climático como ENOS (El Niño/Oscilação Sul) (Sartori, 2000).

No Estado, há quatro grandes províncias geomorfológicas: a Planície Costeira, o Escudo Sul-rio-grandense, a Depressão Periférica e o Planalto, cada uma com características distintas. O Planalto ocupa a metade norte e uma porção no sudoeste do RS, e se subdivide em 7 regiões fisiográficas, sendo o Planalto Médio uma delas.

O relevo dessa região é suave ondulado e bastante homogêneo, com predomínio de solos do tipo Latossolos, Nitossolos e Argilossolos, nas 
áreas mais acidentadas em relevo forte ondulado são encontrados Cambissolos e Neossolos (Streck et al., 2008).

Estas áreas mais acidentadas e com rios de maior vazão são elementos que propiciam a instalação de PCHs, como por exemplo a bacia do rio Ivaí que apresenta uma amplitude de aproximadamente $300 \mathrm{~m}$.
Em vistorias mensais ao local de estudo verifica-se que na porção montante dos reservatórios há intensa exploração agrícola. Estas acompanham o calendário agrícola, o que ocasiona carregamento de materiais para os reservatórios em determinadas épocas do ano onde os solos ficam expostos à ação da intempérie sendo susceptíveis a ação das chuvas.

\section{MATERIAIS E MÉTODOS}

\section{Fonte de dados: dados de TSS e dados de Reflectância}

\section{Total de Sólidos Suspensos}

Para a determinação dos sólidos em suspensão foram coletadas amostras nos três barramentos em estudo. A determinação do total de sólidos em suspensão em nível de laboratório se deu conforme descrito em Wachholz (2011). Para a filtragem em laboratório utilizou-se filtros de celulose de $0,45 \mu \mathrm{m}$ e diâmetro de $47 \mathrm{~mm}$, os quais foram secados previamente por 24 horas em estufa a uma temperatura de $50^{\circ} \mathrm{C}$, a fim de eliminar a umidade. Posteriormente a secagem pesou-se os filtros em balança analítica com acurácia de $0,0001 \mathrm{~g}$ para obtenção do peso inicial (Pi).

Após a realização da filtragem, colocou-se novamente os filtros na estufa na mesma temperatura e tempo de permanência utilizado para a obtenção do peso inicial, (onde permaneceram por 24 horas em $50^{\circ} \mathrm{C}$ novamente). Esses filtros passaram ao processo de pesagem na mesma balança, obtendo-se o peso final $(P f)$. A partir disso foi possível determinar o TSS na unidade $\mathrm{mg} / \mathrm{L}$ para cada amostra com a equação utilizada por Wachholz (2011):

$$
T S S=[P f-P i / V] \times 1000
$$

Onde: TSS - Total de Sólidos em Suspensão; Pf - Peso Final (g); Pi - Peso Inicial (g); V Volume (L).

\section{Reflectância da água - Landsat 8}

A interpretação do comportamento espectral da água em relação aos demais alvos naturais é de grande diferença e complexidade, pois a energia refletida pela água é consideravelmente menor que os demais alvos, sendo o fator de maior relevância para estudos por produtos do sensoriamento remoto. A interpretação dos dados torna-se mais complexa pela interferência atmosférica na região de maior penetração de luz na água pela transmitância variável e alta em função das diferentes profundidades pela presença dos componentes opticamente ativos da água que apresentam espectros semelhantes e a reflectância da superfície da água que é mais elevada quando afetada por outros fatores, como por exemplo, sólidos em suspensão que são melhores estudados com a banda do vermelho (Kirk, 1994, Tzortziou et al., 2006; Park et al., 2014; Sari et al., 2015).

As imagens de satélite do Landsat 8 foram utilizadas para obtenção dos valores de reflectância nos pontos amostrais. Considerando que o Landsat 8 apresenta 11 bandas espectrais e um período de 16 dias de revisita, foi utilizada apenas a banda 4 .

$\mathrm{O}$ processamento das imagens foi realizado utilizando-se o programa Environment for Visualizing Imagens - ENVI versão 4.7. O produto utilizado foi o Superface Reflectance, já georreferenciado e corrigido com valores em reflectância. A banda 4 do Landsat 8 tem comprimento de onda central de $655 \mathrm{~nm}$ correspondente ao vermelho e resolução espacial de $30 \mathrm{~m}$. A banda do vermelho (banda 4) foi utilizada devido esta faixa espectral apresentar melhor resposta a presença de sólidos em suspensão (Kirk, 1994).

Para extração dos valores de reflectância foram criados ROIs (Regiões de Interesse onde é possível selecionar amostras de um raster), nos três reservatórios em análise e utilizados nas imagens selecionadas, totalizando 12 pontos amostrais.

A figura 1 apresenta as datas de aquisição de dados de campo e as respectivas imagens utilizadas. As imagens do mês de julho não 
puderam ser utilizadas devido a quantidade de nuvens sobre a área de estudo. O intervalo de tempo entre a data das imagens de satélite e os dados de campo foi de aproximadamente 1-2 dias, portanto, alguma discrepância é esperada devido ao tamanho dos reservatórios e também a variabilidade temporal e espacial associada com o pulso de sedimentos e a vazão do rio.

Figura 2: Datas dos dados de campo e imagens selecionadas

\begin{tabular}{|l|c|c|c|}
\hline & Mês & Data do trabalho de campo & Data das imagens \\
\hline $\mathbf{1}$ & Junho & $17-06-2015$ & $15-06-2015$ \\
\hline $\mathbf{2}$ & Agosto & $28-08-2015$ & $27-08-2015$ \\
\hline $\mathbf{3}$ & Setembro & $14-09-2015$ & $12-09-2015$ \\
\hline $\mathbf{4}$ & Outubro & $06-10-2015$ & $05-10-2015$ \\
\hline
\end{tabular}

\section{Dados de Precipitação Pluviométrica}

Os dados de precipitação pluviométrica utilizados para essa pesquisa são administrados pela Cooperativa Agropecuária de Júlio de Castilhos-COTRIJUC, que utiliza estes dados para pesquisas em agricultura de precisão e confrontados com dados coletados nas PCHs. O ponto de coleta é a Estação de Ivaí, localizada na área de estudo.

\section{Fatiamento das Imagens}

Para geração das imagens de reflectância da água definiram-se as classes dos valores de reflectância de acordo com o valor mínimo e máximo encontrado no corpo de água da banda selecionada em todas as datas utilizadas $(0,0$ e $9,5<$ respectivamente $)$ totalizando 20 classes.

Após o fatiamento aplicou-se a máscara correspondente ao limite dos reservatórios.

\section{Análise de Regressão}

A estatística descritiva permite de forma sistemática organizar, descrever, analisar e interpretar dados oriundos de estudos ou experimentos, realizados em qualquer área do conhecimento (Pires et al., 2011). O modelo para estimativa da concentração do total de sólidos em suspensão foi desenvolvido com base na análise estatística.

\section{RESULTADOS E DISCUSSÕES}

Os métodos tradicionais de monitoramento de sedimentos em rios baseiam-se em amostras coletadas in situ, a partir de locais prédeterminados. Sabe-se da precisão destes métodos, mas também que o custo e o tempo fazem com que essas distribuições de amostras a serem coletadas sejam limitadas (Ritchie et al., 1987; Ritchie e Shiebe, 2000). Além disso, Montanher et al. (2014), afirmam que geralmente as amostragens são realizadas em locais onde há facilidade de acesso, e não por representatividade espacial da amostra o que pode ocasionar alguma diferença envolvendo a variabilidade espacial e temporal do sedimento em suspensão. Desta forma, dados de sensoriamento remoto podem ser usados para estimar com êxito a concentração de sedimentos na água. Essa estimativa baseia-se na relação entre concentração de sedimentos e a reflêctancia da água. Apesar da baixa frequência de amostragem espacial e temporal, é possivel verificar uma alta correlação entre os valores de TSS e Reflectância. Ritchie; Schiebe \& Mchenry (1976) afirmam que a relação entre sedimentos suspensos e reflectância é nãolinear. Trabalhos desenvolvidos por Kun Shi et al. (2015) e Lin et al. (2012) apontam o modelo exponencial como o de melhor ajuste.

Após avaliar diferentes modelos matemáticos verificou-se que o modelo exponencial foi o que apresentou o melhor ajuste entre os dados de TSS e reflectância no rio Ivaí. Neste sentido, a estimativa através do modelo matemático exponencial apresentou uma forte correlação apresentando um $\mathrm{R}^{2}=0,84$ e um RMS= 11,85 mg/L (Figura 3).

Os valores estimados de TSS mostraram-se coerentes com os valores avaliados in loco, no entanto, em alguns meses, como por exemplo, no mês de junho, ocorrem algumas discordâncias. Tian et al. (2014) afirmam que é esperada essa diferença devido à variabilidade 
temporal e espacial associada com a pluma d'água e a vazão do rio. Pois conforme Kun Shi et al. (2015), a distribuição dos sedimentos em reservatórios é influenciada pelo recebimento de materiais de seus tributários, o que pode ser rapidamente alterada caso os sólidos suspensos e outras matérias particuladas sejam advindos em maior quantidade em eventos de chuva e consequente elevação do fluxo dependendo da dimensão longitudinal do reservatório.

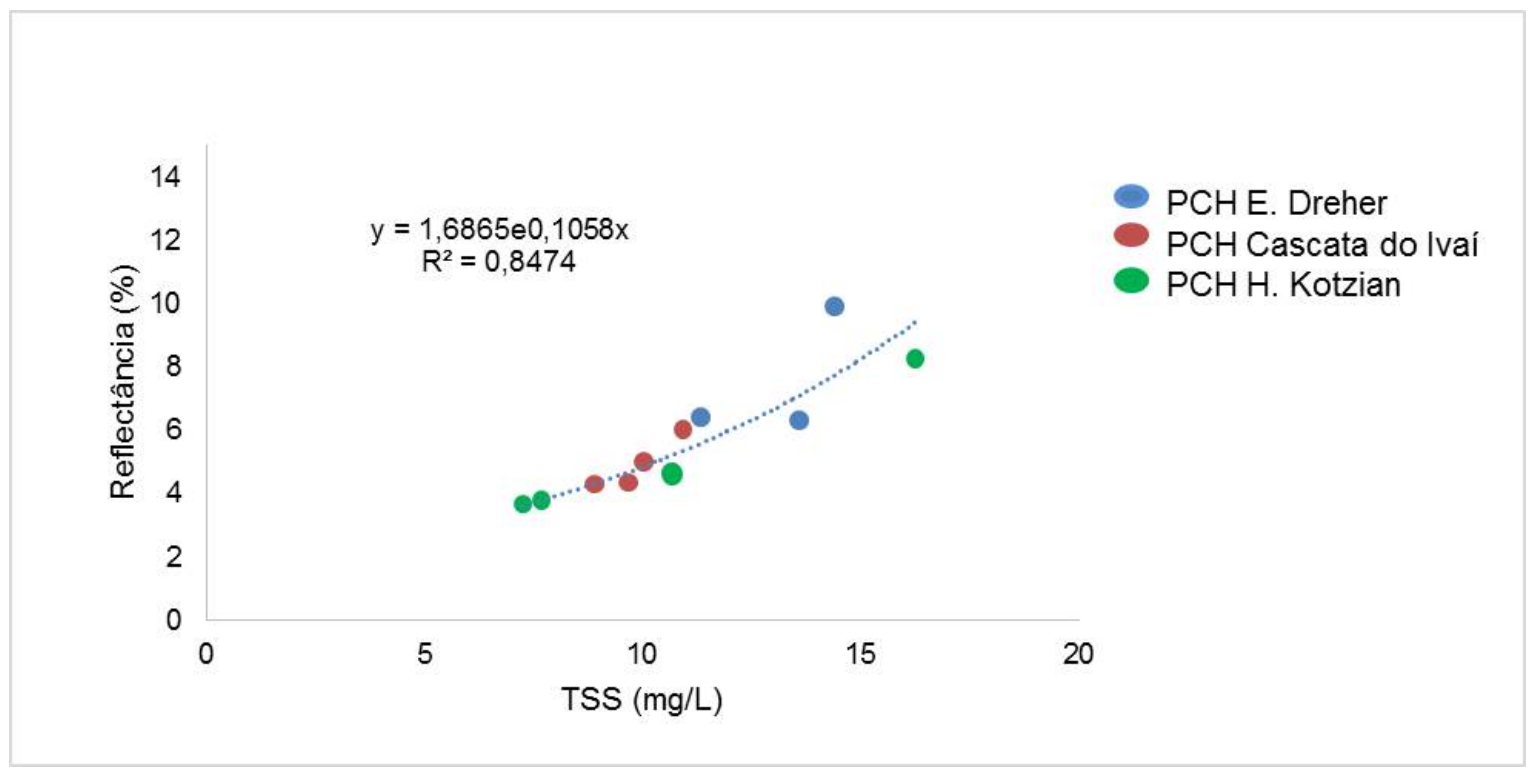

Figura 3 - Gráfico das correlações entre dados de TSS e reflectância.

A figura 4 permite identificar os três barramentos nas imagens estimadas, mostrando uma distribuição semelhante onde o TSS diminui ao longo do rio.

Com base na Figura 3, e considerando que o pico da reflectância se deu na banda 4 (vermelho) no mês de junho verificou-se uma concentração semelhante dos reservatórios E. Dreher e $\mathrm{H}$. Kotzian. Neste mês, foram encontrados os mais elevados valores de reflêctancia e também valores mais elevados de TSS, todos maiores que 13 mg/L. Trabalho realizado por Tian et al. (2014), aponta diferenças entre resultados estimados em concentrações maiores que $10 \mathrm{mg} / \mathrm{L}$, o que podem justificar a diferença do resultado.

No entanto, a precipitação pluviométrica foi de $315 \mathrm{~mm}$ na região das PCHs, ocasionando o transporte de sedimentos para os reservatórios. Desta forma, os sedimentos suspensos podem ser indicadores de possíveis problemas na bacia de captação sujeita a exixtência de áreas com solo exposto devido a agricultura intensa.

$\mathrm{Na}$ análise de agosto, o resultado foi o que se apresentou mais próximo a imagem reflectância. Foram encontrados compartimentos aquáticos e a tendência de deposição dos sedimentos a cada barramento. Os valores pluviométricos não foram muito elevados no local, no entanto um dia antes da passagem do sensor a precipitação local foi de
$42 \mathrm{~mm}$, o que pode ter ocasionado a entrada de água com sedimentos.

No mês de setembro o resultado da imagem estimada de TSS foi de uma água mais homogênea no três setores de análise, indicando uma massa d água com pouco sólidos suspensos, no entanto a precipitação foi de apenas $9 \mathrm{~mm} 2$ dias antes da passagem do sensor, fator este que poderá ter ocasionado a homogeneidade das águas, porém um acumulado de $193 \mathrm{~mm}$ em 30 dias. No mês de outubro, o TSS medido em campo ficou em torno de 10 a $12 \mathrm{mg} / \mathrm{L}$, no entanto, o TSS estimado variou em 10 a $15 \mathrm{mg} / \mathrm{L}$, sendo possível verificar que o modelo criado pode ser aplicado para estimar o TSS.

Considerando os meses amostrados, observouse que o desempenho das imagens Landsat-Oli é diferenciado em função da estimativa da concentração de TSS. Os padrões de sólidos em suspensão estimados mostraram uma distribuição da concentração semelhante com a imagem ao longo do rio, diminuindo no sentido montantejusante, mostrando a tendência de deposição dos sedimentos devido ao efeito cascata das PCHs. Os altos valores de reflectância e consequentemente de TSS podem estar associados aos elevados índices pluviométricos na região sul devido ao efeito do El Niño. 


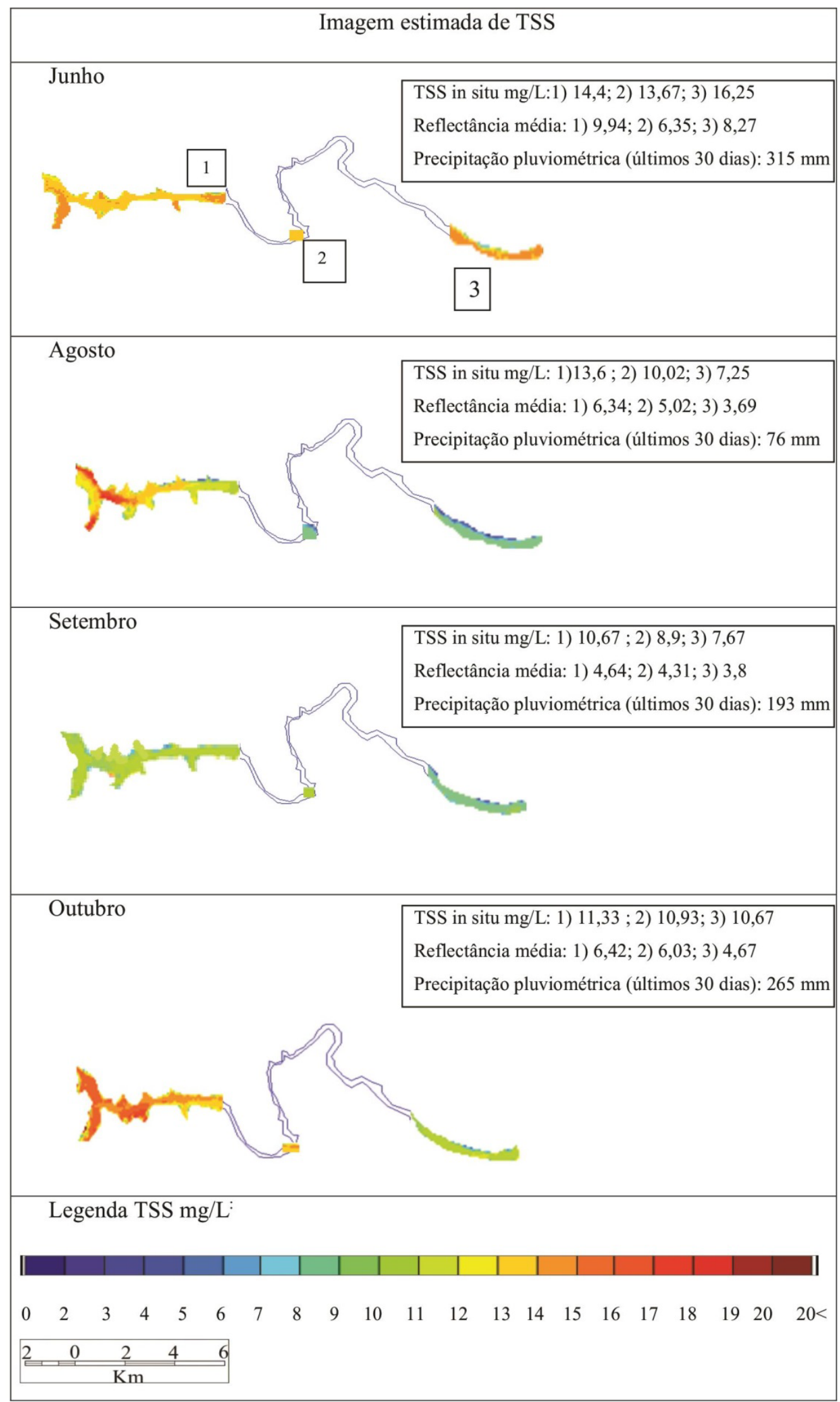

Figura 4 - Identificação dos três barramentos nas imagens estimada de TSS.

\section{CONSIDERAÇÕES FINAIS}

No caso estudado a atenção maior deve se impactos podem ser proporcionalmente maiores dar pelo efeito cascata, implantação de diversas dos causados por grandes obras, principalmente PCHs em sequência em um mesmo rio, pois os no que diz respeito ao transporte de sedimentos 
provocando diversas consequências, entre elas, alteração da vazão do rio.

Nesse cenário de pequenos barramentos o modelo proposto mostrou-se indicado para a estimativa da concentração de sólidos suspensos. O ideal é que se utilize o período de um ano de monitoramento para a construção dessa curva de ajuste. Porém o objetivo proposto foi atingido com resultado satisfatório, onde o fatiamento das imagens de reflectância e das imagens estimadas foi muito semelhante, evidenciando a deposição dos sedimentos a cada barramento.

Além disso, a abordagem realizada aqui pode ser aplicada em outras regiões com semelhantes características do rio e da bacia. Mas deve-se levar em consideração que a relação entre a reflectância por satélite e as concentrações encontradas de TSS podem variar para outros reservatórios.

\section{AGRADECIMENTOS}

Os autores agradecem a Capes pela concessão da bolsa de estudo e a empresa Coprel -RS, pela disponibilidade em realizar a pesquisa.

\section{REFERÊNCIAS}

BRASIL. Resolução da ANEEL, N ${ }^{\circ}$ 394, DE 4 DE DEZEMBRO DE 1998. Estabelece os critérios para o enquadramento de empreendimentos hidrelétricos na condição de pequenas centrais hidrelétricas. Agência Nacional de Energia Elétrica. Disp. em: http://www.aneel.gov.br/cedoc/res19983 94.pdf. Acesso em: 15 out. 2016.

CHEN, Z.; CURRAN, P.J; HANSOM, J.D. Derivative Reflectance Spectroscopy to Estimate Suspended Sediment Concentration. Remote Sensing of Environment, v. 40, p. 67-77, 1992.

CMSAPAR- Participações S.A BME Energia. Disp. em: http://www.cmsapar.com.br/bme.php?lang=pt. Acessado em: 03/01/2017.

COTRIJUC- Cooperativa Agropecuária de Júlio de Castilhos. Disp. em: http://www.cotrijuc.com.br/site/ cotrijuc.php?mod=chuvas\&cat=ver\&nucleo_id=1\&pag=4 Acesso em: 10-11-2015

KIRK, J.T.O. Ligth and photosynthesis in aquatic ecosystems. 2ed. Cambridge University Press: New York, 1994.

MONTANHER, O.C.; BARBOSA, C.C.F.; RENNÓ, C.D, SILVA, T.S.F. Empirical models for estimating the suspended sediment concentration in Amazonian white water rivers using Landsat 5/TM. International Journal of Applied Earth Observation and Geoinformation, v. 29, p. 67-77, 2014.

OLIVEIRA, A.S. Interação entre sistemas frontais na América do Sul e a convecção da Amazônia. São José dos Campos, 1986. 115p. Dissertação (Mestrado em Meteorologia), Instituto Nacional de Pesquisas Espaciais.

PARK, E. \& LATRUBESSE, E. Modeling suspended sediment distribution patterns of the Amazon River using MODIS data. Remote Sensing of Environment. v. 147, p. 232-242, 2014.

PIRES, C.A.F. Geoestatística: caderno didático. Santa Maria: UFSM-CCNE, 2011.

RITCHIE, J.C.; FRANK, R.; SCHIEBE, J.; MCHENRY, R. Remote Sensing of Suspended Sediments in Surface Waters. Photogrammetric Engineering and Remote Sensing, v. 42, p. 1539-1545, 1976.

RITCHIE, J.C.; COOPER, C.M.; YONGQING, J. Using Landsat Multispectral Scanner data to estimate suspended sediments in Moon Lake, Mississippi. Remote Sensing of Environment, v. 23, n. 1, p. 65-81, 1987.
RITCHIE, J.C. \& SHIEBE, F.R. Water quality. In: SHULTZ, G.A., ENGMAN, E.T (Eds.), Remote Sensing in Hydrology and Water Management. Springer, p. 287-303, 2000.

VANESSA, S.; NILZA, C.; MASATO, K. Estimativa da concentração de sedimentos suspensos com sensores ópticos: revisão. Revista Brasileira de Recursos Hídricos, v. 20, p. 816-836, 2015.

SARTORI, M.G.B. As variações pluviométricas e o regime das chuvas na Região Central do Rio Grande do Sul. Boletim de Geografia Teorética. v. 45-46, n. 23, p.70-84, 1993.

SARTORI, M.G.B. Clima e Percepção. São Paulo, 2000. 227p. Tese (Doutorado em Geografia), Universidade Estadual de São Paulo.

KUN,S.; YUNLIN, Z.; GUANGWEI, Z; XIAOHAN, L.; YONGQIANG, Z.; HAI, X.; BOQIANG, Q; GE, L.; YUNMEI, L. Long-term Sremote monitoring of total suspended matter concentration in Lake Taihu using $250 \mathrm{~m}$ MODIS-Aqua data Remote. Sensing of Environment, v. 164, p. 43-56, 2015.

STRECK, E.V. Solos do Rio Grande do Sul. 2. ed. Porto Alegre: EMATER, 2008.

TIAN, L.; WAI, O.W.H; CHEN, X.; LIU, Y.; FENG, L.; LI, J.; HUANG, J. Assessment of Total Suspended Sediment Distribution under Varying Tidal Conditions in Deep Bay: Initial Results from HJ-1A/1B Satellite CCD Images. Remote Sensing, v. 6, n. 10, p. 9911-9929, 2014.

TZORTZIOU, M.; SUBRAMANIAM, A.; HERMAN, J.R.; GALLEGOS, C.L.; NEALE, P.J.; HARDING JR., L.W. Remote sensing reflectance and inherent optical properties in the mid Chesapeake Bay. Estuarine, Coastal and Shelf Science, v. 72, p. 16-32, 2007.

WACHHOLZ, F. Influência das bacias hidrográficas e caracterização espaço-temporal de variáveis limnológicas em reservatório no Rio Grande do Sul. Rio Claro, 2011. 191p. Tese (Doutorado em Geografia), Universidade Estadual Paulista.

WANG, J.J. \& LU, X.X. Estimation of suspended sediment concentrations using Terra MODIS: An example from the Lower Yangtze River, China. Science of the Total Environment, v. 408, p. 1131-1138, 2010.

Submetido em 12 de junho de 2016 Aceito em 25 de agosto de 2017 